changed pesticide-use pattern. The ecotoxicological and toxicological effects linked with the various scenarios of pesticide use will be compared quantitatively with the aid of "environmental indicators." In addition, other effects of the altered pesticide use, including ecology and biodiversity will be taken into account.

The final goal is to provide a comprehensive overview of the effects associated with altered pesticide use in genetically modified crops. Such an overview may serve as a tool for policy makers in their risk-benefit analysis for the introduction of genetically modified crops in the environment.

For more information, contact the Task Group Chairman Gijs A. Kleter $<$ g.a.kleter@rikilt.dlo.nl>.

\section{www.iupac.org/projects/2001/2001-024-2-600.html}

\title{
Provisional Recommendations
}

\section{IUPAC Seeks Your Comments}

Provisional recommendations are drafts of IUPAC recommendations on terminology, nomenclature, and symbols made widely available to allow interested parties to comment before the recommendations are finally revised and published in Pure and Applied Chemistry.

\section{Recommendation for the Naming of Element of Atomic Number 110}

A joint IUPAC-IUPAP Working Party (JWP) has confirmed the discovery of element number 110 with the collaboration of Hofmann et al from the Gesellschaft für Schwerionenforschung $\mathrm{mbH}$ (GSI) in Darmstadt, Germany. In accord with IUPAC procedures, the discoverers have proposed a name and symbol for the element. The Inorganic Chemistry Division Committee now recommends this proposal for acceptance. The proposed name is darmstadtium with symbol Ds. This proposal lies within the long established tradition of naming an element after the place of its discovery. The full text is available online, see link below.

\section{Comments by 30 June 2003 to:}

Prof. John Corish

University of Dublin

Chemistry Department, Trinity College

Dublin 2, Ireland

Tel.: + [353] (1) 6081776

E-mail: jcorish@tcd.ie

\section{www.iupac.org/reports/provisional/abstract03/corish_300603.html}

\section{Definitions of Terms Relating to Reactions of Polymers and to Functional Polymeric Materials}

This document defines the terms most commonly encountered in the field of polymer reactions and functional polymers. The scope has been limited to terms that are specific to polymer systems. The document is organized into three sections. The first defines the terms relating to reactions involving and specific to polymers. Names of individual chemical reactions are omitted from the document, even in cases where the reactions are important in the field of polymer reactions. The second section defines the terms relating to polymer reactants and reactive polymeric materials. The third section defines terms describing functional polymeric materials.

\section{Comments by 31 July 2003 to:}

Prof. Máximo Barón

Universidad de Belgrano

Facultad de Ciências Exactas y Naturales

Villanueva 1324

Buenos Aires, 1426

Argentina

Tel.: +[54] 1145114700

Fax: +[54] 1148214887

E-mail: baron@ub.edu.ar

www.iupac.org/reports/provisional/abstract03/horie_310703.html 